\title{
DATABASE MANAGEMENT FOR FACIAL RECOGNITION SYSTEM
}

\author{
Sk. Naveed ${ }^{1}$ \\ ${ }^{1,}$ Department of Computer Science and Engineering, Koneru Lakshmaiah Education Foundation, \\ Vaddeswaram-522502, Guntur, AP, India
}
N.Ramya $\mathbf{a}^{2}$
${ }^{2}$ Department of Computer Science and Engineering, Koneru Lakshmaiah Education Foundation, Vaddeswaram-522502, Guntur, AP, India
D.Manasa ${ }^{3}$
${ }^{3}$ Department of Computer Science and Engineering, Koneru Lakshmaiah Education Foundation, Vaddeswaram-522502, Guntur, AP, India

\author{
N. Ramya Sri ${ }^{4}$ \\ ${ }^{4}$ Department of Computer Science and Engineering, Koneru Lakshmaiah Education Foundation, \\ Vaddeswaram-522502, Guntur, AP, India
}

Article DOI: https://doi.org/10.36713/epra5585

\begin{abstract}
The face is one of the easiest ways to differentiate the individual identity of each other. Face recognition is a personal identification system that uses personal characteristics or facial features of a person to identify the person's identity. The most used human face recognition process is face detection, where this procedure takes place very quickly in humans, except under certain conditions where the object is located at close distance. The purpose of this project is to develop face recognition based automated student attendance system. In order to achieve high quality performance, the test images and training images of this proposed approach are limited to frontal and upright facial images that consist of a single face only. The test images and training images have to be captured by using the same device to ensure no quality difference. In addition, the students have to register in the database to be recognized. The enrolment can be done on the spot through the user-friendly interface.
\end{abstract}




\section{EPRA International Journal of Research and Development (IJRD)

\section{INTRODUCTION}

The enhancement of science and technology leads to make the life more comfortable than older days. The emerging technologies like neutrosophic shortest path $[1,2,3,4,5]$, transportation problem $[6,7,8]$, uncertainty problem $[9,10,11,12,13,14]$, fuzzy shortest path $[15,16,17,18,19]$, PowerShell [20], wireless sensor network $[21,22,23,24,25,26,27,28]$, computer language [29,30], neural network [31], routing [32] making the products more intelligent and self-healing based. The smart city applications like smart water, smart grid, smart parking, smart resource management, etc. are based on IoT and IoE $[33,34,35,36]$ technologies. We have the development available to us to enable the organization of our consistently lives and the sharing of significant information with our associates, families and others. Why development is huge in our consistently life. It is a basic contraption that we can't avoid, it has a huge impact in the vast majority of our lives Technology fundamentally handles the instruments, advancements and strategies used to help us with dealing with issues and simply improve our everyday schedules and easier to encounter to a great extent [37,38]. Advancement is inevitable in our normal everyday presences [39]. This is in light of the fact that presence without advancement is senseless in the present incredible world. Development, which joins instruments to propel unforeseen development, use and information exchange, has as its basic objective of making tasks easier and the handling of various issues of mankind. Right when development advances and makes our continues with extensively more worthwhile, we should pressure that it is so useful to our lives.

Face recognition is the process of identifying an already detected object as a known or unknown face. Face recognition technology is gradually evolving to a global biometric technique since it requires zero effort from the user end while compared with other biometric methods. In spite of the fact that other techniques of identification such as fingerprints, or iris scanning can be more accurate, face recognition has always remains a major focus of research because of its non-invasive nature and because it is people's basic method of person identification. The purpose of this project is to develop the process of face recognition based automated student attendance system. Commonly, student's attendances are taken manually by using attendance register given by the faculty members in class, which is a time consuming event. Moreover, it is very difficult to check one by one student in a large classroom environment with distributed branches whether the authenticated students are actually responding or not. So, why not make it automated quickly and much efficient.

\section{DIGITAL IMAGE IN DATABASE}

Face recognition basically requires a database to store a number of pictures. Human face recognition is based on face detection because in order to identify a person's in a picture, face recognition method has to locate the people's face in the picture. Human face recognition compares the similarity between the captured pictures of the student with the pictures of the student within the database and finds the most similar students. It is processed by capturing the video of the students, convert it into frames, and relate it with the database to ensure their presence or absence, mark attendance to the particular student to maintain the records.

There are three major steps:

1: To find a good database of faces with multiple images for each individual.

2. To detect faces in the database images and use them to train the face recognizer.

3. Test the face recognizer to recognize faces it was trained for.

To perform the process of face detection, there must be a database which consists of student images in gif format. There will be 11 images for each individual .In each image, each individual student has a different facial expression like happy, sad, normal, surprised, sleepy etc. 


\section{EPRA International Journal of Research and Development (IJRD)}

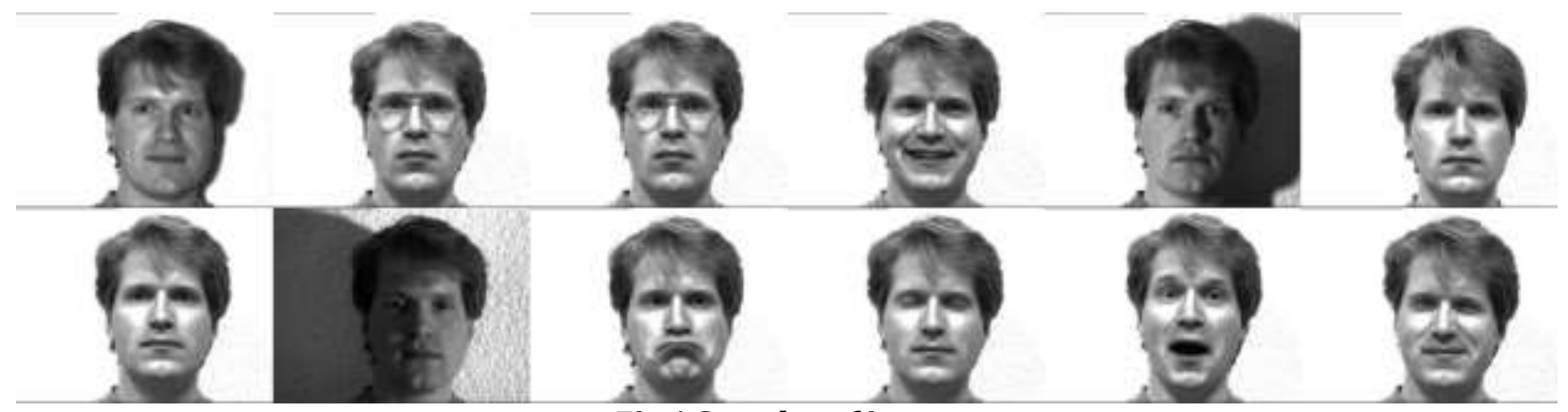

Fig.1 Samples of image

We will use database by using to store different number of images of the total images of each individual student in training our face recognizer and the single image of each student individual to test our face recognition algorithm.

They are different types of image processing

1. Low level

2. Medium level

3. High level

Low level processing means performing main operations on images such as Scanning an image resize, rotate image, RGB to gray level conversion etc , The output of an image obtained after low level processing is fresh image. Medium level processing means extracting regions of interest from output of low level processed image. It deals with identification of edges .This process is also called as segmentation. High level processing deals with adding of artificial intelligence to medium level processed signal.

\section{ELEMENTS OF DIGITAL IMAGE PROCESSING}

The basic operations performed in a digital image processing are:

- Acquisition

- Storage

- Processing

- Communication

- Display

\section{CONCLUSION}

The aim of this Project is to capture the video of the students, convert it into frames, relate it with the database to ensure their presence or absence, mark attendance to the particular student to maintain the record.

\section{REFERENCES}

1. Broumi, S., Dey, A., Talea, M. et al. Shortest path problem using Bellman algorithm under neutrosophic environment. Complex \& Intelligent Systems, 5 (2019), 409-416.

2. Kumar, R., Dey, A., Broumi, S., and Smarandache, F. A study of neutrosophic shortest path problem. In Neutrosophic Graph Theory and Algorithms. IGI Global, 2020.

3. Kumar, R., Edalatpanah, S. A., Jha, S., Broumi, S., Singh, R., and Dey, A. A multi objective programming approach to solve integer valued neutrosophic shortest path problems. Neutrosophic Sets and Systems, 24 (2019), 139151.

4. Kumar, R., Edalatpanah, S. A., Jha, S., and Singh, $R$. A novel approach to solve gaussian valued neutrosophic shortest path problems. International Journal of Engineering and Advanced Technology, 8 (2019), 347-353.

5. Kumar, R., Edaltpanah, S. A., Jha, S., Broumi, S., and Dey, A. Neutrosophic shortest path problem. Neutrosophic Sets and Systems, 23 (2018), 5-15.

6. Pratihar, J., Kumar, R., Dey, A., and Broumi, S. Transportation problem in neutrosophic environment. In Neutrosophic Graph Theory and Algorithms. IGI Global, 2020.

7. Kumar, R., Edalatpanah, S. A., Jha, S., and Singh, R. A Pythagorean fuzzy approach to the transportation problem. Complex \& Intelligent Systems, 5 (2019), 255-263.

8. Pratihar, J., Kumar, R., Edalatpanah, S. A., and Dey, A. Modified Vogel's approximation method for transportation problem under uncertain environment. Complex \& Intelligent Systems (2020), 1-12.

9. Gayen, S., Jha, S., Singh, M., and Kumar, R. On a generalized notion of anti-fuzzy subgroup and some characterizations. International Journal of Engineering and Advanced Technology, 8 (2019), 385-390.

10. Gayen, S., Smarandache, F., Jha, S., and Kumar, $R$. Interval-valued neutrosophic subgroup based on interval-valued triple t-norm. In Neutrosophic Sets in Decision Analysis and Operations Research. IGI Global, 2020. 


\section{SJIF Impact Factor: 7.001| ISI I.F.Value:1.241| Journal DOI: 10.36713/epra2016 ISSN: 2455-7838(Online) EPRA International Journal of Research and Development (IJRD)}

11. Gayen, S., Smarandache, F., Jha, S., Singh, M. $K$. , Broumi, S., and Kumar, R. Introduction to plithogenic subgroup. In Neutrosophic graph Theory and Algorithms. IGI Global, 2020.

12. Gayen, S., Smarandache, F., Jha, S., Singh, M. K., Broumi, S., and Kumar, R. Soft Subring Theory Under Interval-valued Neutrosophic Environment. Neutrosophic Sets and Systems, 36 (2020), 16.

13. Gayen, S., Smarandache, F., Jha, S., and Kumar, $R$. Introduction to interval-valued neutrosophic subring. Neutrosophic Sets and Systems, 36 (2020), 17.

14. Gayen, S., Smarandache, F., Jha, S., Singh, M. $K$., Broumi, S., and Kumar, R. Introduction to plithogenic hypersoft subgroup. Neutrosophic Sets and Systems, 33 (2020), 208-233.

15. Kumar, R., Edalatpanah, S. A., and Mohapatra, $H$. Note on "Optimal path selection approach for fuzzy reliable shortest path problem ". Journal of Intelligent \& Fuzzy Systems (2020), 1-4.

16. Kumar, R., Jha, S., and Singh, R. A different approach for solving the shortest path problem under mixed fuzzy environment. International Journal of Fuzzy System Applications, 9 (2020), 132-161.

17. Kumar, R., Jha, S., and Singh, R. Shortest path problem in network with type-2 triangular fuzzy arc length. Journal of Applied Research on Industrial Engineering, 4 (2017), 1-7.

18. Kumar, Ranjan, Edalatpanah, S. A., Jha, Sripati, Gayen, Sudipta, and Singh, Ramayan. Shortest path problems using fuzzy weighted arc length. International Journal of Innovative Technology and Exploring Engineering, 8 (2019), 724-731.

19. Kumar, R., Edalatpanah, S. A., Jha, S., Gayen, S., and Singh, R. Shortest path problems using fuzzy weighted arc length. International Journal of Innovative Technology and Exploring Engineering, 8 (2019), 724-731.

20. Mohapatra, H., Panda, S., Rath, A. K., Edalatpanah, S. A., and Kumar, R. A tutorial on powershell pipeline and its loopholes. International Journal of Emerging Trends in Engineering Research, 8 (2020), 975-982.

21. Mohapatra, H., Rath, S., Panda, S., and Kumar, $R$. Handling of man-in-the-middle attack in wsn through intrusion detection system. International Journal of Emerging Trends in Engineering Research, 8 (2020), 1503-1510.

22. Mohapatra, H., Debnath, S., and Rath, A. K. Energy management in wireless sensor network through eb-leach. International Journal of Research and Analytical Reviews, 56 (2018), 5661.

23. Mohapatra, H., Rath, A. K., Landge, P. B., Bhise, D., Panda, S., and Gayen, S. A comparative analysis of clustering protocols of wireless sensor network. International Journal of Mechanical and Production Engineering Research and Development, 10 (2020), 8371-8386.

24. Mohapatra, H. and Rath, A. K. A survey on fault tolerance based clustering evolution in wsn. IET Networks (2020).

25. Mohapatra, H., Debnath, S., Rath, A. K., Landge, P. B., Gayen, S., and Kumar, R. An efficient energy saving scheme through sorting technique for wireless sensor network. International Journal of Emerging Trends in Engineering Research, 8 (2020), 4278-4286.

26. Mohapatra, H. and Rath, A. K. Fault tolerance in wsn through uniform load distribution function. International Journal of Sensors, Wireless Communications and Control, 10 (2020).

27. Mohapatra, H. and Rath, A. K. Fault tolerance through energy balanced cluster formation (ebcf) in wsn. In Smart Innovations in Communication and Computational Sciences ( 2018), springer, 313-321.

28. Mohapatra, Hitesh and Rath, Amiya Kumar. Fault tolerance in WSN through PE-LEACH protocol. IET Wireless Sensor Systems, 9, 6 (Dec. 2019), 358-365(7).

29. Mohapatra, H. C Programming: Practice. Amazon, 2018.

30. Mohapatra, H. and Rath, A. K. Fundamentals of Software Engineering. BPB, 2020.

31. Mohapatra, H. HCR by Using Neural Network. 2009.

32. Panda, M., Pradhan, P., Mohapatra, H., and Barpanda, N. K. Fault tolerant routing in heterogeneous environment. International Journal of Scientific \& Technology Research, 8 (2019), 1009-1013.

33. Mohapatra, H. Offline drone instrumentalized ambulance for emergency situations. International Journal of Robotics and Automation, 9 (2020), 251-255.

34. Mohapatra, H. and Rath, A. K. Detection and avoidance of water loss through municipality taps in india by using smart tap and ict. IET Wireless Sensor Systems (2019), https--doi.

35. Panda, H., Mohapatra, H., and Rath, A. K. Wsnbased water channelization: an approach of smart water. In Smart Cities: Opportunities and Challenges. Springer, Singapore, 2020.

36. Rout, S. S., Mohapatra, H., Nayak, R. K., Tripathy, R., Bhise, D., Patil, S. P., and Rath, A. $K$. Smart water solution for monitoring of water usage based on weather condition. International Journal of Engineering and Technical Research, 8 (2020), 5335-5343. 